\title{
Raman Spectroscopy for Quantitative Analysis of Point Defects and Defect Clusters in Irradiated Graphite
}

\author{
Keisuke Niwase \\ Hyogo University of Teacher Education, Hyogo 673-1494, Kato, Japan \\ Correspondence should be addressed to Keisuke Niwase, niwase@hyogo-u.ac.jp \\ Received 16 July 2011; Accepted 11 October 2011 \\ Academic Editor: Maher S. Amer \\ Copyright () 2012 Keisuke Niwase. This is an open access article distributed under the Creative Commons Attribution License, \\ which permits unrestricted use, distribution, and reproduction in any medium, provided the original work is properly cited.
}

We report the development of Raman spectroscopy as a powerful tool for quantitative analysis of point defect and defect clusters

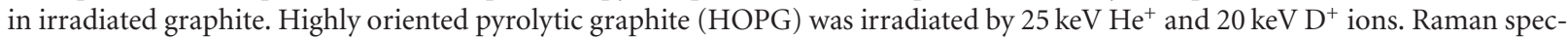
troscopy and transmission electron microscopy revealed a transformation of irradiated graphite into amorphous state. Annealing experiment indicated a close relation between Raman intensity ratio and vacancy concentration. The change of Raman spectra under irradiation was empirically analyzed by "disordered-region model," which assumes the transformation from vacancycontained region to disordered region. The model well explains the change of Raman spectra and predicts the critical dose of amorphization, but the nature of the disordered region is unclear. Then, we advanced the model into "dislocation accumulation model," assigning the disordered region to dislocation dipole. Dislocation accumulation model can simulate the irradiation time dependencies of Raman intensity ratio and the $c$-axis expansion under irradiation, giving a relation between the absolute concentration of vacancy and Raman intensity ratio, suggesting an existence of the barrier on the mutual annihilation of vacancy and interstitial.

\section{Introduction}

Graphite is one of useful materials for thermal nuclear and fusion reactors due to their outstanding nature with respect to heat load, neutron activation, and so forth. Since the fire of Windscale nuclear reactor occurred in 1957 [1] due to a spontaneous release of stored energy from irradiated graphite, radiation damage has been recognized to be important problems to be solved for irradiated graphite. Also, dimensional changes of growth parallel to $c$-axis and contraction within the basal planes decrease in $c$-axis electrical resistivity and so forth occur as serious problems. In spite of a lot of investigations on irradiated graphite [2-33], the natures of point defects and defect clusters, and their reaction kinetics are obscure. This can be attributed to the anisotropy of the graphite lattice which requires a description of the motion of interstitials and vacancies in terms of activation energies parallel and perpendicular to the basal planes, and the instability of vacancy and interstitial clusters $[3,7]$.

Raman spectroscopy has been used for the characterization of graphite and investigations on the graphitization process and on the change of graphite structure under irradiation. The advantages of this method for the examination of graphite are a selective sensitiveness to the structural changes in the basal plane, such as crystalline size or the formation of a single vacancy or its clusters, and extensive and continuous sensitivity over the range from ordered to amorphized graphite.

In this paper, we review our experimental $[8-10,12$, $15]$ and theoretical studies $[18,19,26,29]$ on irradiated graphite. Our study started from the discovery of irradiationinduced amorphization of graphite, which was judged from the change of TEM diffraction pattern observed along the $c$-axis [8]. The TEM specimens were investigated by microRaman spectroscopy, leading us to directly compare the change of the TEM and the related Raman results [9]. Important Raman results were obtained by annealing of irradiated HOPG at relatively low temperatures from $373 \mathrm{~K}$ to $873 \mathrm{~K}$. Relaxation of Raman intensity ratio started to appear at comparatively low temperature of $373 \mathrm{~K}$, which implies a close relationship between Raman intensity ratio and vacancy concentration [10]. 

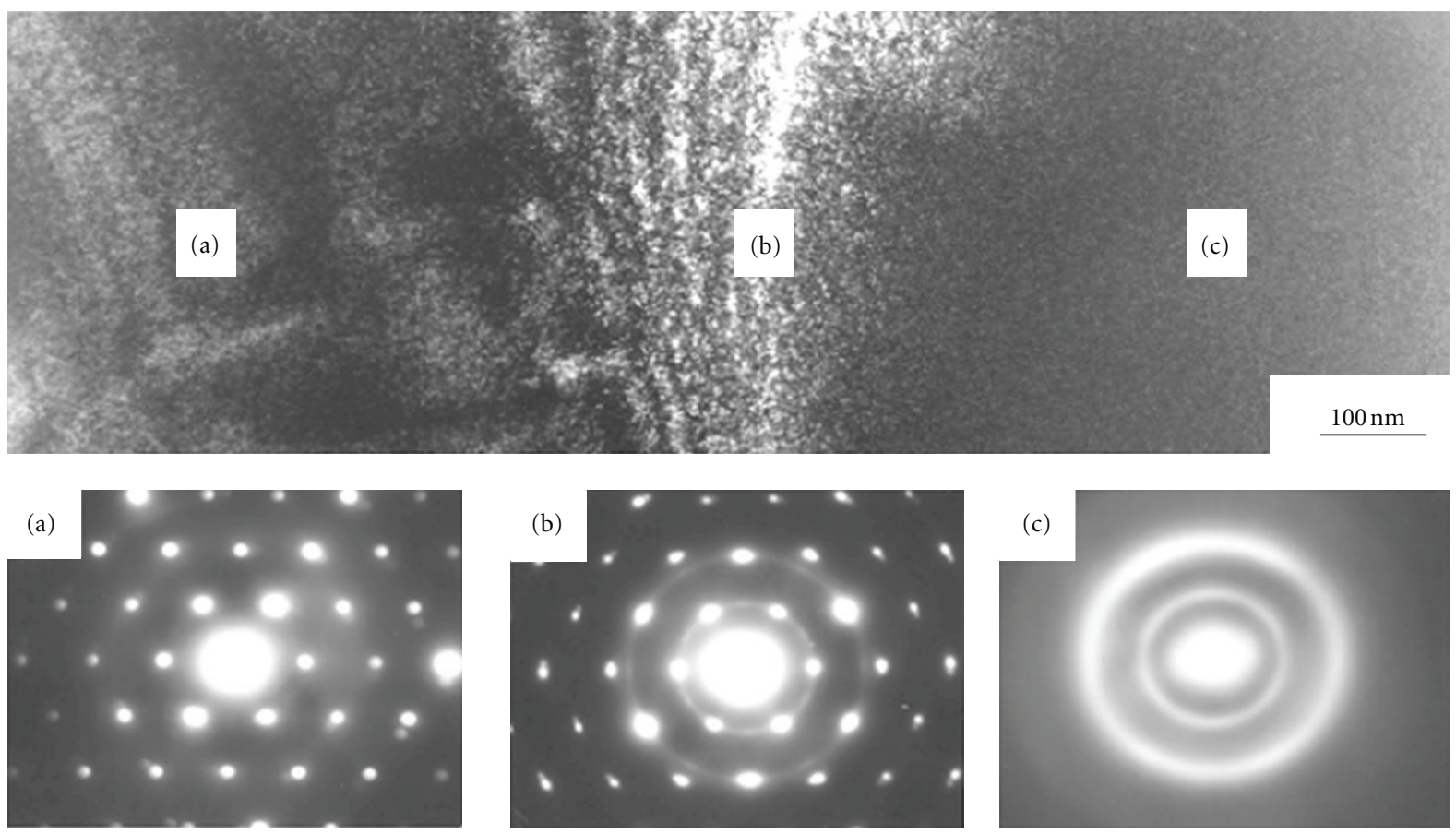

FIGURE 1: TEM image of highly oriented pyrolytic graphite (HOPG) irradiated with $20 \mathrm{keV} \mathrm{D}^{+}$at $473 \mathrm{~K}$. The left hand side area (a) of the image is a part of the specimen covered by a mesh wire and the right hand side area (c) is a part irradiated to a dose of $2.5 \times 10^{21}$ ions $/ \mathrm{m}^{2}$. Selected area diffraction patterns corresponding to the areas (a), (b) and (c) are given below the photograph.

We firstly proposed a semiempirical model of "disordered-region model" to explain the dose and the temperature dependencies of the change of Raman spectra under irradiation $[18,19]$. The model assumes the transformation from vacancy-contained region to disordered region and well explains the change of Raman spectra, predicting the critical dose of amorphization. However, the disorderedregion model cannot predict the change of the concentration of point defects and defect clusters under irradiation nor give information on the structure of the disordered region. Then, we proposed an advanced model of "dislocation accumulation model," which can simulate the square-root time dependence of Raman intensity ratio under irradiation and gives a relation between the absolute concentration of vacancy and the Raman intensity ratio, suggesting an existence of the barrier on the mutual annihilation of vacancy and interstitial [26]. Thus, we show Raman spectroscopy as a powerful tool for the study of radiation damage of graphite.

\section{Experimental Results for Irradiated Graphite}

\subsection{Ion Irradiation to HOPG Foils}

2.1.1. TEM Results and Raman Spectra. Thin foils of highly oriented pyrolytic graphite for transmission electron microscopy (TEM) were irradiated with $25 \mathrm{keV} \mathrm{He}^{+}$and $20 \mathrm{keV} \mathrm{D}^{+}$at temperatures ranging from room temperature (RT) to $973 \mathrm{~K}$ [8]. Figure 1 shows the TEM images and associated diffraction patterns of $20 \mathrm{keV} \mathrm{D}^{+}$-irradiated HOPG foil. The left-hand side area, marked with (a), was covered by a mesh wire, and, then, there remains the original graphite structure, as confirmed by the [0001] diffraction pattern of a graphite crystalline lattice shown underneath. The right hand side area, (c), irradiated with a dose of $2.5 \times$ $10^{21} \mathrm{D} / \mathrm{m}^{2}$, on the other hand, completely loses the extinction contours and coarse dotty contrast, only halo ring being seen in the diffraction pattern. The midst side area, (b), irradiated with an intermediate dose shows a mixed diffraction pattern, spots, and halo rings. Thus, initially perfect crystalline graphite gradually loses atomic ordering with increasing dose and becomes amorphous when the implanted dose is above a critical value. The critical dose for the amorphous becomes larger with the increase of the irradiation temperature.

The amorphization, characterized by the disappearance of the white dotty contrast in the dark field image and the transition from the spots to the halos in the diffraction pattern, is also observed in the case of $\mathrm{He}^{+}$irradiation. The appearance of the [0001] diffraction pattern of the irradiated specimens in Figure 1 was classified into three patterns of spot, halo and spot, and halo, which are referred as $s, h s$, and 


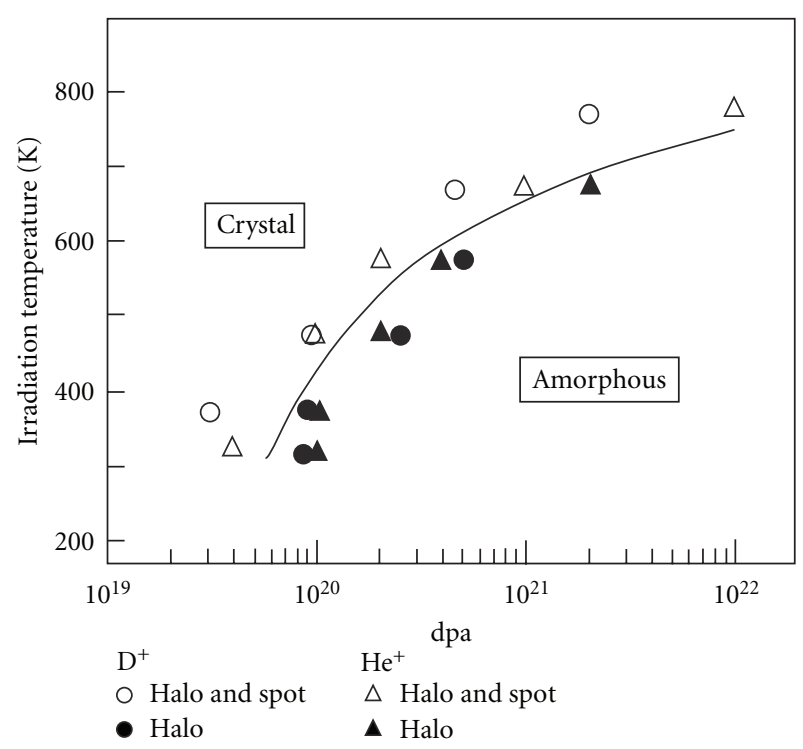

Figure 2: Conditions of appearance of TEM diffraction patterns of $h$ s (halo and spot) and $h$ (halo) by $20 \mathrm{keV} \mathrm{D}{ }^{+}$and $25 \mathrm{keV} \mathrm{He}^{+}$ irradiation. The critical conditions of amorphization are indicated by the solid curve. Note that the critical dpa for the $\mathrm{D}^{+}$irradiation almost coincides with that for the $\mathrm{He}^{+}$irradiation.

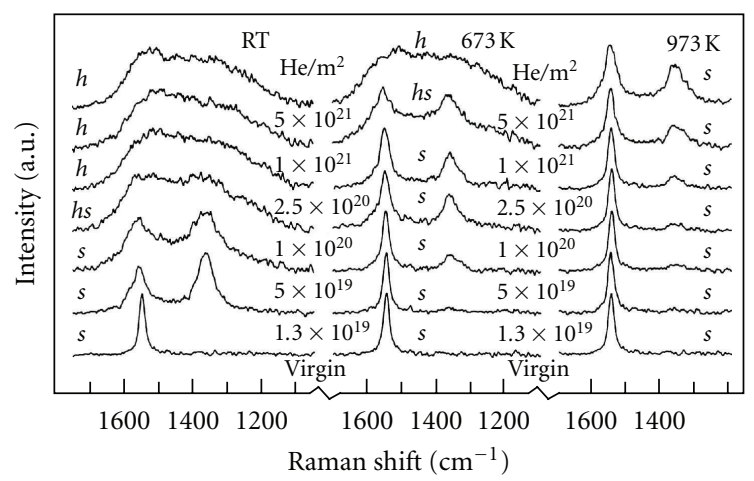

Figure 3: Changes in Raman spectra for HOPG foils irradiated with $25 \mathrm{keV} \mathrm{He}^{+}$at three different temperatures of RT, 673, and $973 \mathrm{~K}$. The appearance of the TEM diffraction pattern of the foils is denoted by $s$ (spot), $h s$ (halo and spot), and $h$ (halo).

$h$, respectively and hereafter. The critical dose of $\mathrm{He}^{+}$irradiation is much less than that of the $\mathrm{D}^{+}$irradiation. Nevertheless, the calculated value of the critical dpa (displacement per atom) for the amorphization by the $\mathrm{He}^{+}$irradiation was the same as that for the $\mathrm{D}^{+}$irradiation, as shown in Figure 2. This indicates that the amorphization is basically controlled by an atomic displacement and the difference in the nature of the incident particles or the chemical effect plays a minor role.

The TEM specimens were investigated by micro-Raman spectroscopy with a resolution of $2 \mu \mathrm{m}$. Figure 3 compares the changes of Raman spectra of HOPG foils irradiated with $25 \mathrm{keV} \mathrm{He} e^{+}$at RT and 673 and $973 \mathrm{~K}$. The spectrum before irradiation is given at the bottom of the figures, where the Raman-active $E_{2 g}$ mode can only be found at $1580 \mathrm{~cm}^{-1}$. After irradiation, a new peak appears around
$1355 \mathrm{~cm}^{-1}$, which can be assigned as the maximum phonon density of states, caused by the relaxation of the wave-vector selection rule. The appearances of the [0001] diffraction pattern investigated with TEM for each specimen are referred as $s, h s$, and $h$, respectively, in Figure 3 . One can see that the Raman spectral change closely correlates with the TEM diffraction change. The onset of amorphization denoted by $h s$ corresponds to the onset of remarkable broadening of the two main Raman peaks, while the completion of amorphization denoted by $h$ corresponds to a complete overlapping of the two peaks. The effect of irradiation on Raman spectra becomes less appreciable with increasing the irradiation temperature, because of dynamic annealing during irradiation. Increasing the irradiation temperature is seen to delay the onset of amorphization. Contrary to the close relation between the broadening of the Raman peak width and amorphization checked by TEM diffraction pattern, the reduction of the crystalline size, which is calculated from the intensity ratio of $I_{1355} / I_{1580}$ [34], does not coincide with the amorphization process investigated by TEM.

\subsubsection{Diagram for the Change of Amorphization. Raman} spectra are deconvoluted into several characteristic peaks with a least-square algorithm. The spectra of the irradiated graphite denoted by $s$ and $h s$ are decomposed into four Lorentzian peaks two dominant Lorentzian peaks at about 1355 and $1580 \mathrm{~cm}^{-1}$ and additional two minor Lorentzian peaks at about 1200 and $1500 \mathrm{~cm}^{-1}$, while the spectra denoted by $h$ cannot be fitted well by the Lorentizans, but can so by Gaussians.

Figures 4(a) and 4(b) show the changes of the intensity ratio of the two peaks $\left(I_{1355} / I_{1580}\right)$ and a full width at half maximum of the $1580 \mathrm{~cm}^{-1}$ peak $\left(\mathrm{FWHM}_{1580}\right)$, determined by the fitting analysis, against the dose. A difference can be clearly observed between the two. Below $473 \mathrm{~K}$, the $I_{1355} / I_{1580}$ is already larger than 1 at the initial stage of irradiation. After reaching a maximum, it gradually decreases and levels off. On the other hand, the FWHM 1580 gradually increases at the initial stage, but it increases dramatically when amorphization starts. The final stage of amorphization, denoted by $h$, corresponds to a value of $180 \mathrm{~cm}^{-1}$. Thus the change of the FWHM 1580 more closely correlates with the amorphization process than that of $I_{1355} / I_{1580}$.

Figure 5 shows how the $\mathrm{FWHM}_{1580}$ changes in relation to the dose. Linear relationships can be seen at each irradiation temperature, but the increase rate decreases with increasing irradiation temperature. A significant reduction of the increase rate can be found between 573 and $673 \mathrm{~K}$.

Figure 6 shows the relation between the $\mathrm{FWHM}_{1580}$ and the $I_{1355} / I_{1580}$ under $25 \mathrm{keV} \mathrm{He}^{+}$irradiation. The three linear relations can be seen to correspond to the appearance of the TEM diffraction patterns of $s, h s$, and $h$. One should note, however, that several data below $473 \mathrm{~K}$ deviated from this relation, indicating different paths from the solid line to the dashed one.

2.1.3. Annealing of Irradiated Graphite. An annealing study was done using Raman spectroscopy in order to examine thermal stability of the defect structures in the HOPG foils 


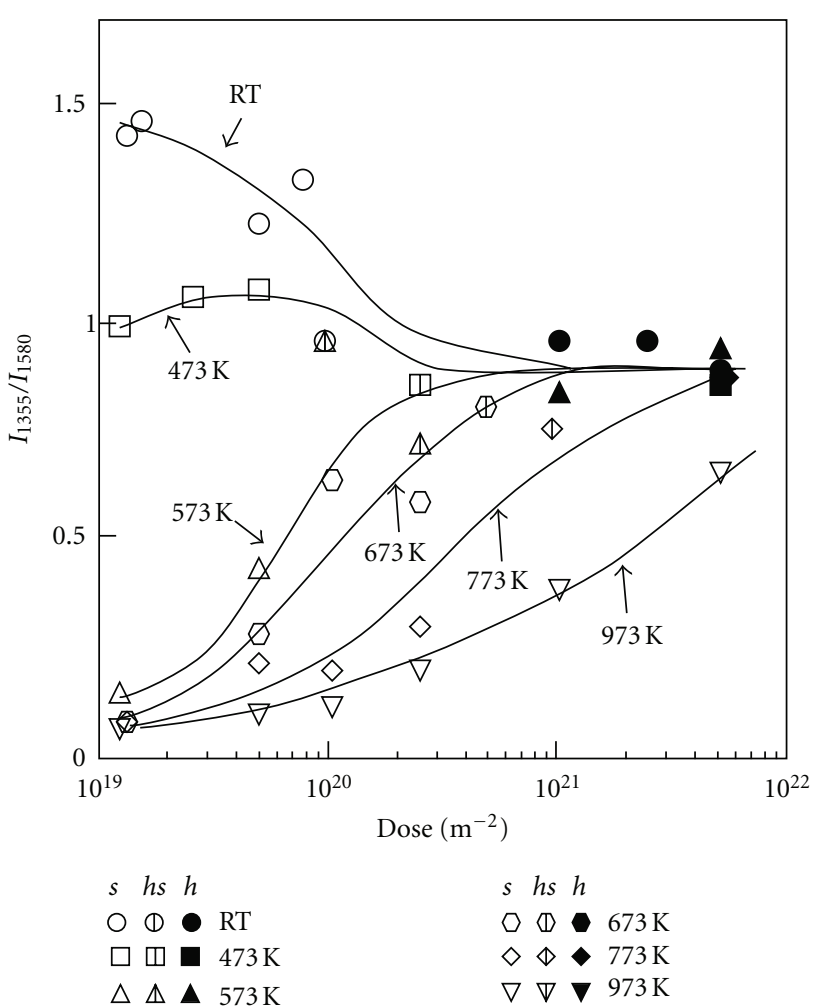

(a)

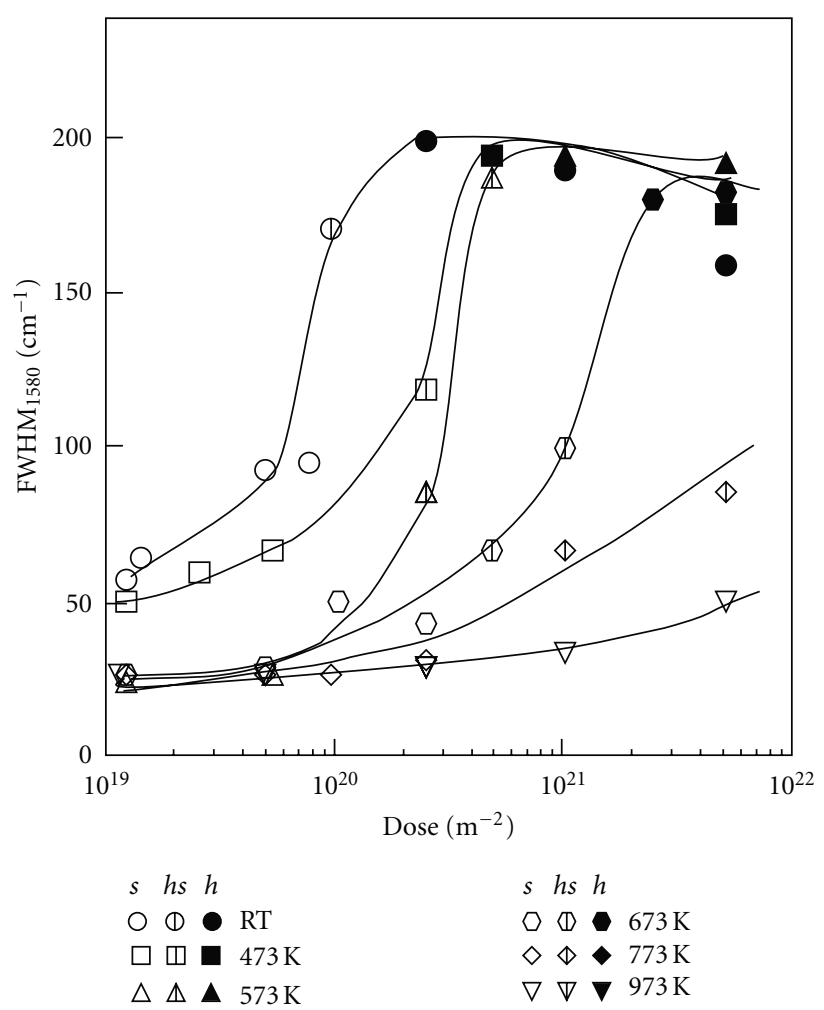

(b)

FIgUre 4: (a) The intensity ratio of $I_{1355} / I_{1580}$ and (b) the peak width of $1580 \mathrm{~cm}^{-1}$ versus the dose in a logarithmic scale for HOPG foils irradiated with $25 \mathrm{keV} \mathrm{He}^{+}$at temperatures ranging from RT to $973 \mathrm{~K}$. The appearance of the TEM diffraction pattern of the foils is denoted by $s$ (spot), $h s$ (halo and spot), and $h$ (halo). The solid curves are guides for eyes.

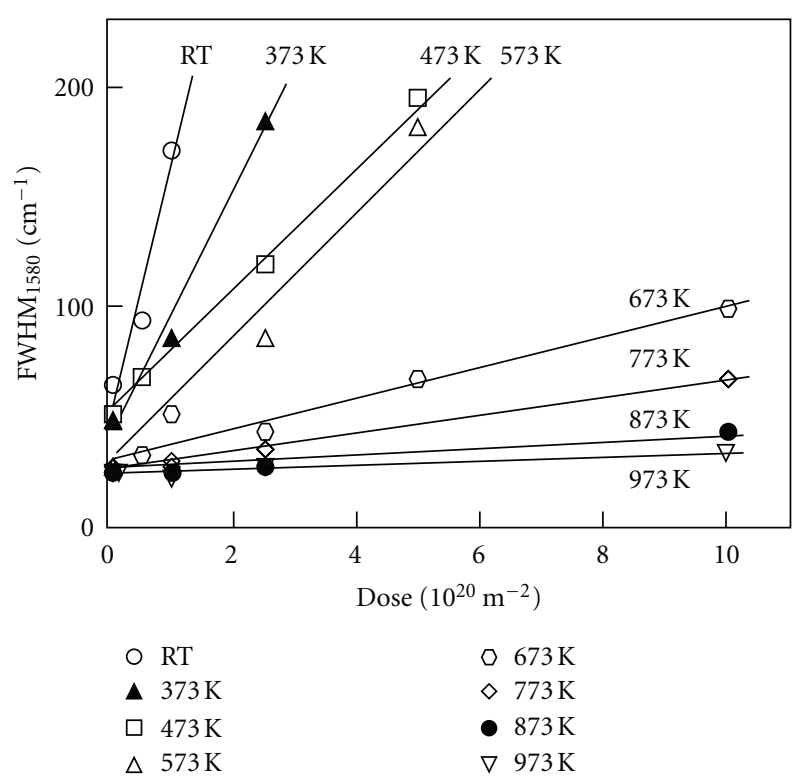

FIGURe 5: Peak width of $1580 \mathrm{~cm}^{-1}$ versus the dose in a linear scale for HOPG foils irradiated with $25 \mathrm{keV} \mathrm{He}^{+}$at temperatures ranging from RT to $973 \mathrm{~K}$. The dose range corresponds to the amorphizing stage. The peak widths are seen to linearly increase against the dose. The solid lines are guides for eyes. irradiated with $20 \mathrm{keV} \mathrm{D}^{+}$at room temperature and the result is shown in Figure 7. The appearances of the TEM diffraction pattern investigated before annealing are denoted by $s, h s$, and $h$. One can clearly distinguish the annealing effect between the unamorphized HOPG $(s)$ and amorphized one (h). For the unamorphized HOPG $(s)$ a remarkable recovery of the Raman spectra appears at annealing temperatures between 373 and $573 \mathrm{~K}$, whereas only a slight change can be seen in the amorphized HOPG $(h)$.

\subsection{HRTEM Image and Raman Spectra of Neutron-Irradiated} Graphite Fiber. A combined study of high-resolution TEM and Raman spectroscopy for neutron-irradiated graphite fibers has given important information on the nature of irradiation-produced defects [12]. Raman spectrum for a dose of $1.4 \times 10^{23} \mathrm{n} / \mathrm{m}^{2}$ shows a sharp and intense damage peak at $1355 \mathrm{~cm}^{-1}$, whereas that for $1.9 \times 10^{24} \mathrm{n} / \mathrm{m}^{2}$ is very broad, as respectively shown in Figures $8(\mathrm{c})$ and $8(\mathrm{~d})$. The two spectra correspond well to those of the ion irradiated HOPG before amorphization $(s)$ and in amorphizing process (hs), respectively. In spite of the appearance of sharp damage peak in the Raman spectra, the corresponding lattice image in Figure 9 still keeps the long-range order of the basal planes and does not show an apparent reduction of the crystalline 


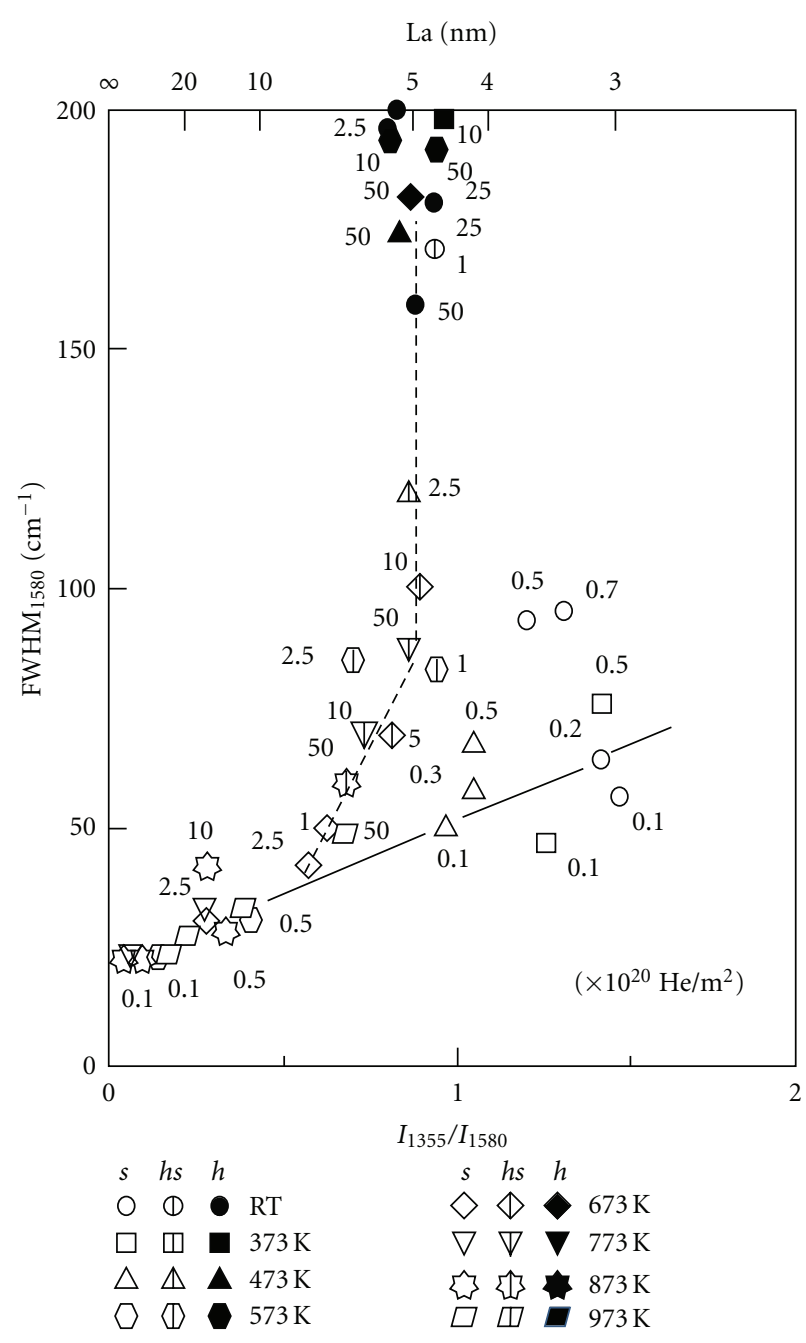

FIgURE 6: Peak width of $1580 \mathrm{~cm}^{-1}$ versus intensity ration of $I_{1355} / I_{1580}$ for HOPG foils irradiated with $25 \mathrm{keV} \mathrm{He} \mathrm{He}^{+}$at temperatures ranging from RT to $973 \mathrm{~K}$. Open, half-closed, and closed symbols, respectively, correspond to the appearance of the TEM diffraction patterns of $s$ (spot), $h s$ (halo and spot), and $h$ (halo). The in-plane crystalline size La calculated by Tuinstra and Koenig's relation [34] is also indicated in the figure. The calculated La, especially for the data along the solid line, should not be attributed to the in-plane crystalline size but to the phonon correlation length broken by in-plane defects of vacancies, as described in the text.

size. Instead, it shows many fine contrasts in the lattice fringe which probably come from the production of in-plane defects. This means that the increase of $I_{1355} / I_{1580}$ is not necessarily owing to the reduction of so-called "crystalline size La" but owing to the production of in-plane defects such as vacancies. Correspondingly, the significant reduction of the intensity of the damage peak at $1355 \mathrm{~cm}^{-1}$ for the unamorphized HOPG (s) on the annealing below $573 \mathrm{~K}$ in Figure 7 can be well explained by the mutual annihilation of vacancies with mobile interstitials.

The above considerations lead us to conclude that the increase of $I_{1355} / I_{1580}$ in the early stage of the irradiation is originated from the irradiation produced in-plane defect

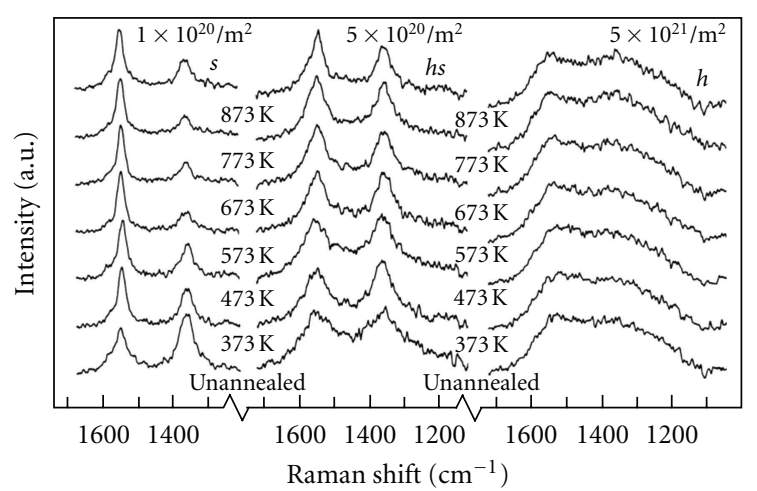

FIGURE 7: Changes in Raman spectra on isochronally annealing in steps of $100 \mathrm{~K} / 1800 \mathrm{~s}$ for HOPG foils irradiated with $25 \mathrm{keV} \mathrm{D}^{+}$at RT to three different doses. The appearance of the TEM diffraction patterns of the foils before the annealing is denoted by $s$ (spot), $h s$ (halo and spot), and $h$ (halo).

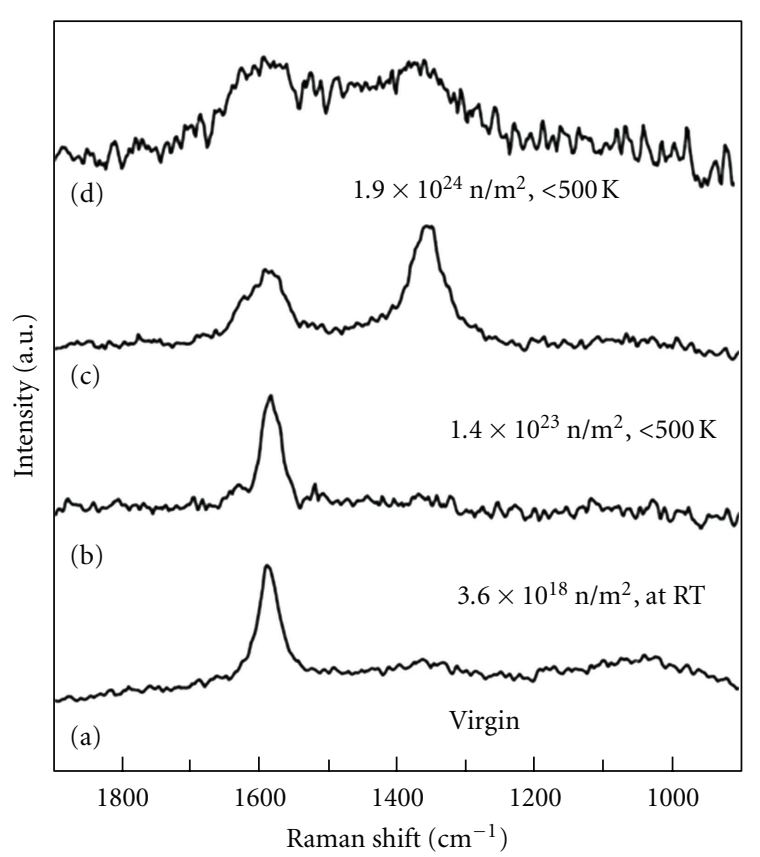

FIGURE 8: Raman spectra of carbon fibers of (a) unirradiated, and irradiated with (b) $14 \mathrm{MeV}, 3.6 \times 10^{18} \mathrm{n} / \mathrm{m}^{2}$, (c) $1 \mathrm{MeV}, 1.4 \times$ $10^{23} \mathrm{n} / \mathrm{m}^{2}$, and (d) $1 \mathrm{MeV}, 1.9 \times 10^{24} \mathrm{n} / \mathrm{m}^{2}$.

such as vacancy itself. This approves the result by Nakamura and Kitajima, who have explained the change of $I_{1355} / I_{1580}$ of phonon correlation length due to the increase of the concentration of irradiation-produced vacancies [13]. Turbulence and disordering of the basal plane, on the other hand, are clearly seen in the lattice image of the fiber, which shows the broad Raman spectrum in Figure 8. The turbulence and disordering should originate from some irregularity of bonding angle and length and cause the broadening of the Raman spectrum which hardly returns to the sharp peak on annealing below $873 \mathrm{~K}$, as shown in Figure 7. 


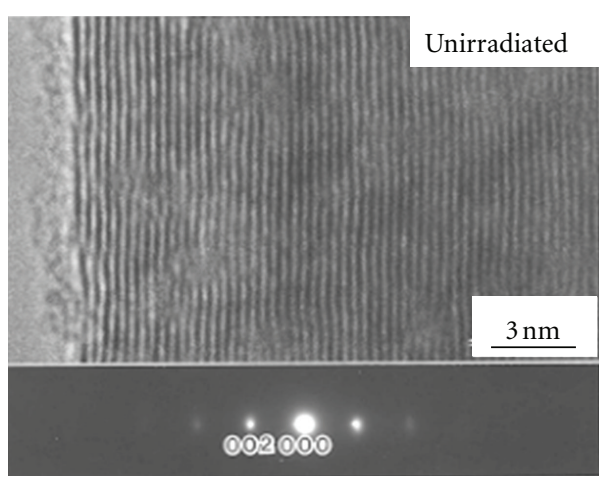

(a)

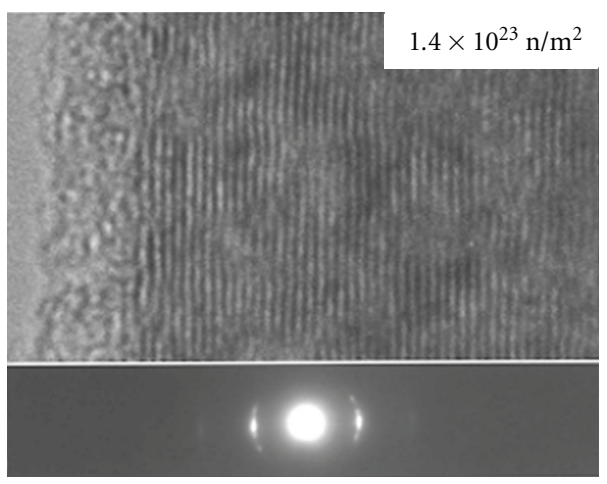

(c)

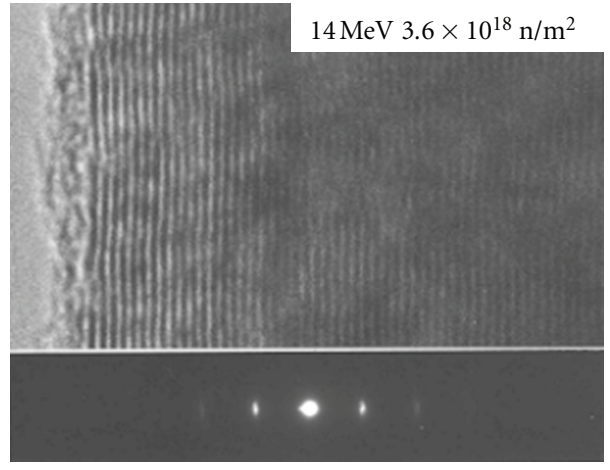

(b)

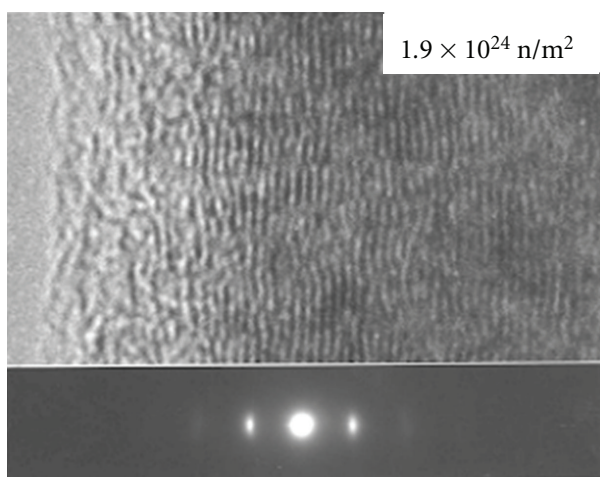

(d)

FIGURE 9: Lattice images and diffraction patterns in high-resolution TEM for carbon fibers of (a) unirradiated, and irradiated with (b) 3.6 $\times 10^{18} \mathrm{n} / \mathrm{m}^{2}$, (c) $1.4 \times 10^{23} \mathrm{n} / \mathrm{m}^{2}$, and (d) $1.9 \times 10^{24} \mathrm{n} / \mathrm{m}^{2}$.

\section{Model for the Change of Raman's Spectra and Irradiation-Induced Amorphization}

3.1. Raman's Spectra and Irradiation-Induced Defects. The intensity ratio of $I_{1355} / I_{1580}$ for various grades of graphite has been widely accepted to have an inverse proportionality against a "crystalline size La" [34]. However, we have found a significant reduction of the $I_{1355} / I_{1580}$ for RT-irradiated HOPG on annealing below $573 \mathrm{~K}$, which cannot be explained by the crystalline size change (Figure 7). Reduction of the intensity ratio on annealing only occurred for unamorphized specimens, which correspond to the ones indicated by the solid line in Figure 6 . We have concluded that the change of the $I_{1355} / I_{1580}$ under irradiation should not be attributed simply to the crystalline size change but to defects produced in the basal planes such as vacancies. Nakamura and Kitajima, on the other hand, performed a real-time measurement of the change of Raman spectra under $\mathrm{He}^{+}$ irradiation and have found that the $I_{1355} / I_{1580}$ is proportional to the square root of irradiation dose [13]. They successfully explained the result by the reduction of phonon correlation length due to vacancy formation, giving a relation between the Raman intensity ratio and the concentration of vacancy $C_{V}$, as follows:

$$
C_{V}=k\left(\frac{I_{1355}}{I_{1580}}\right)^{2},
$$

where $k$ is a constant. By replacing the $C_{V}$ with $C_{V}{ }^{\prime}=k^{-1} C_{V}$, we get

$$
C_{V}{ }^{\prime}=\left(\frac{I_{1355}}{I_{1580}}\right)^{2} .
$$

Here, we define the in-plane defect concentration $C_{V}{ }^{\prime}$, which is expressed by the value of $I_{1355} / I_{1580}$, as the one in Raman intensity units (R.I. units), and use it hereafter.

Figure 10 shows a schematic drawing of the change in the peak width of $1580 \mathrm{~cm}^{-1}$ and the intensity ratio of $I_{1355} / I_{1580}$ on irradiation, in which schematic pictures of graphite layers considered from Raman and HRTEM results are shown. The solid line can be assigned to the formation of in-plane defect such as vacancies, of which existence does not induce the turbulence of the basal planes. The deviation upward from the solid line in the relation between FWHM $_{1580}$ and $I_{1355} / I_{1580}$ (Figure 6) and the appearance of turbulence of the basal plane (Figure 9), on the other hand, corresponds to the start of the amorphization. The values of the $I_{1355} / I_{1580}$ at the deviation points decrease with increasing the irradiation temperature, which are about 1.4 below $373 \mathrm{~K}$, about 1.0 at $473 \mathrm{~K}$, and about 0.5 above $573 \mathrm{~K}$. The decrease of the value should correspond to the decrease of the saturation concentration of vacancy. At temperatures lower than $473 \mathrm{~K}$, the upward deviation from the solid line is seen to start at higher values of $I_{1355} / I_{1580}$ compared to those above $573 \mathrm{~K}$, as shown by the dotted lines. This is probably due to the less 


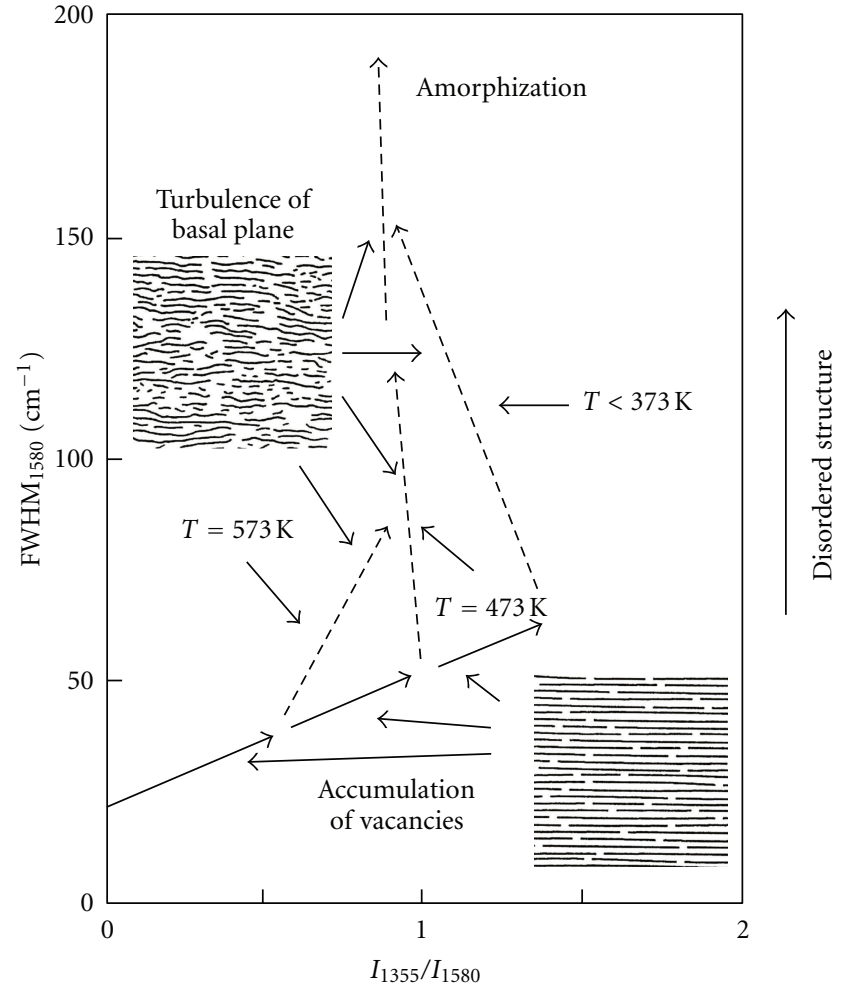

FIGURE 10: Schematic drawing of the change in peak width of $1580 \mathrm{~cm}^{-1}$ and intensity ratio of $I_{1355} / I_{1580}$ on irradiation. Schematic pictures of graphite layers considered from Raman and HRTEM results are shown in the figure. The solid lines can be assigned to the formation of in-plane defects such as vacancies. The upward deviation from the solid line corresponds to the turbulence and disordering of the basal plane. At temperatures lower than $473 \mathrm{~K}$, the upward deviation from the solid line is seen to start at higher values of $I_{1355} / I_{1580}$ compared to those above $573 \mathrm{~K}$, as shown by the dotted lines. This is probably due to the less annihilation of in-plane defects with mobile interstitials.

annihilation of vacancies with mobile interstitials at lower irradiation temperatures.

3.2. Semiempirical Model (Disordered-Region Model). The Raman intensity ratio of $I_{1355} / I_{1580}$ and the peak width of FWHM $_{1580}$ give different information on the formation of vacancies and disordered regions. The change of Raman spectra is now considered with the following assumptions.

(a) Single vacancies are saturated to the concentration of $C_{V S}(T)$ at an irradiation temperature $T$ to a dose $t_{1}$.

(b) Graphite structural regions with single vacancies $(G$ regions) gradually transform into disordered regions ( $D$ regions). The transformation rate is proportional to the saturated concentration of single vacancies $C_{V S}(T)$.

(c) The Raman intensity ratio $\left(I_{1355} / I_{1580}\right)$ and the peak width of the G-peak $\left(\mathrm{FWHM}_{1580}\right)$ are expressed by the sum of Raman components from the $G$ and $D$ regions.

(d) The $I_{1355} / I_{1580}$ has a square-root dependence on the concentration of vacancies and $D$ regions. The FWHM $_{1580}$ has a square-root dependence on the concentration of vacancies, but has a proportional dependence on the concentration of the $D$ regions.

According to (a) and (b), the concentration of the $D$ regions, $C_{D}$, at a dose of $t_{2}$ is given by

$$
C_{D}\left(t_{2}\right)=k C_{V S}(T)\left(t_{2}-t_{1}\right),
$$

where $k$ is a reaction constant. The value of $C_{D}$ before the saturation of the in-plane defects is negligibly small after several repetition of $t_{1}$. From (c) and (d) the intensity ratio and the peak width of the $1580 \mathrm{~cm}^{-1}$ peak at $t_{2}$ are, respectively, expressed as

$$
\begin{aligned}
I_{1355} / I_{1580}\left(t_{2}\right) & =C_{V}\left(t_{2}\right)^{1 / 2}+A C_{D}\left(t_{2}\right)^{1 / 2}, \\
\mathrm{FWHM}_{1580}\left(t_{2}\right) & =E+B C_{V}\left(t_{2}\right)^{1 / 2}+D C_{D}\left(t_{2}\right),
\end{aligned}
$$

where $A, B$, and $C$ are constant parameters and $E$ is the peak width of virgin HOPG at room temperature (Figure 12). One should note that $C_{V}$ and $C_{D}$ are not expressed in atomic concentration, but in R.I. units, which are defined in the last section. From (3)-(5), the FWHM 1580 is expressed as

$$
\begin{aligned}
\text { FWHM }_{1580}\left(t_{2}\right) & \\
= & E+B\left[\frac{I_{1355}}{I_{1580}}\left(t_{2}\right)-A k^{1 / 2} C_{V S}(T)^{1 / 2}\left(t_{2}-t_{1}\right)^{1 / 2}\right] \\
& +D k C_{V S}(T)\left(t_{2}-t_{1}\right) .
\end{aligned}
$$

Now, in comparing the model with experimental results, it is clear that (6) cannot predict the changes of Raman spectra only by itself, as it contains three variable parameters of FWHM $1580\left(t_{2}\right), I_{1355} / I_{1580}\left(t_{2}\right)$, and $C_{V S}(T)$. Then we use the experimental results of $I_{1355} / I_{1580}$ (Figure $4(\mathrm{a})$ ) to give information on the changes of concentration of $C_{V}$ and $C_{D}$ under irradiation. All the constant parameters $k, A, B$, $D$, and $E$ can be definitely obtained from the experimental results to be $0.2,2 / 3,30,200$, and 22 , respectively. With these parameters and the experimental results of $I_{1355} / I_{1580}$, the disordered-region model can predict the dose and temperature dependencies of $\mathrm{FWHM}_{1580}$, using the saturated concentration of single vacancies, $C_{V S}(T)$, as a fitting parameter at each irradiation temperature.

Figure 11 compares the experimental and predicted results of the dose dependence of the $\mathrm{FWHM}_{1580}$ at each irradiation temperature. Symbols represent the experimental results, while solid curves are the calculated results. The theoretical curves closely match the experimental results. The gradual increase of the $\mathrm{FWHM}_{1580}$ before amorphization and the remarkable increase after the onset of amorphization are well predicted. The relation between the $\mathrm{FWHM}_{1580}$ and 


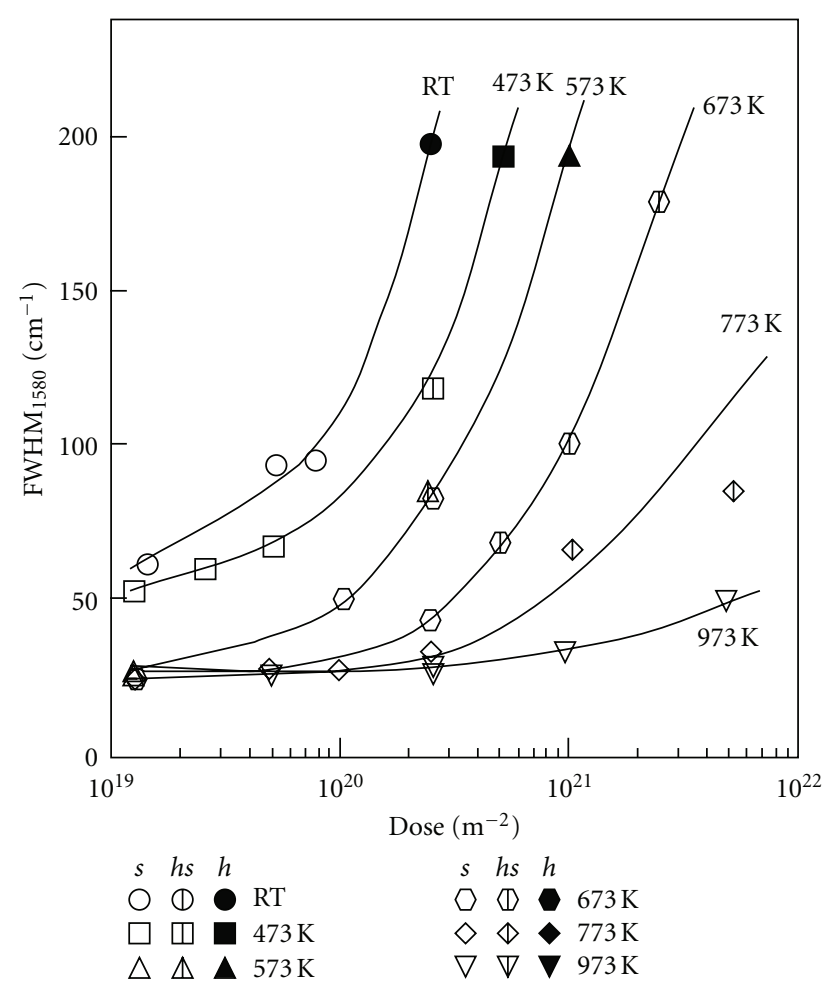

FIGURE 11: Comparison of the peak width of $1580 \mathrm{~cm}^{-1}$ calculated by the present model with the experimental result. The calculated values are shown as solid curves.

the $I_{1355} / I_{1580}$ shown in Figure 6 is also well predicted by the model $[18,19]$.

In this disordered-region model, we can clearly define the critical dose of amorphization $\left(t_{c}\right)$, that is, the dose at which the $D$ regions reach a critical amount of $A$, thereby causing the disappearance of diffraction spots and the appearance of the halo in the TEM diffraction pattern of the irradiated graphite. Using (3) and neglecting the saturation time $t_{1}$ since $t_{1} \ll t_{c}$, the amorphization condition can be expressed as

$$
C_{V S} t_{C}=A^{\prime},
$$

where $A^{\prime}$ is $k^{-1} A$. This indicates that the critical dose of amorphization, $t_{C}$, is inversely proportional to the saturated concentration of single vacancies, $C_{V S}$.

Figure 13 shows the Arrhenius plot of the $C_{V S}{ }^{-1}$, of which value is determined as the fitting parameter in the model calculation. We can find two linear relations, showing two activation energy of $0.083 \mathrm{eV}$ below $573 \mathrm{~K}$ and $0.58 \mathrm{eV} \mathrm{[19]} \mathrm{above} 573 \mathrm{~K}$. It is interesting to compare the change of $C_{V S}{ }^{-1}$ with the TEM diffraction pattern on the amorphization. Notably, two lines parallel to the Raman results can be drawn passing between the two points denoted by $h s$ and $h$ at each temperature, as shown in Figure 13. The consistency of the temperature dependencies of the critical dose of amorphization given by two different methods of Raman spectroscopy and TEM strongly supports the disordered-region model.

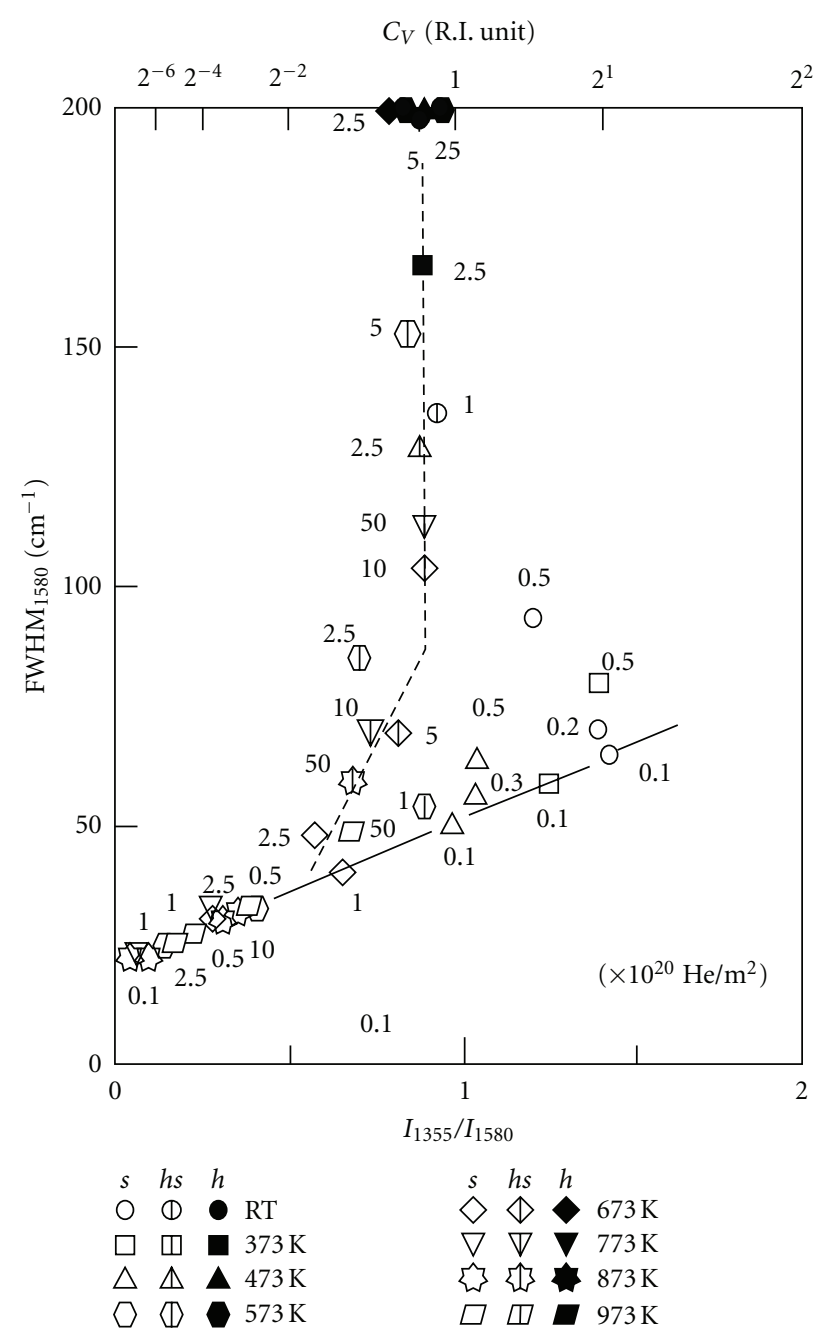

Figure 12: Relation between the peak width of $1580 \mathrm{~cm}^{-1}$ and the intensity ratio of $I_{1355} / I_{1580}$ predicted by the present model. The value of $C_{V}$ are expressed in R.I. units.

3.3. Kinetic Model (Dislocation Accumulation Model). The disordered-region model can predict the temperature dependence of the critical dose for amorphization and give an insight into the mobilities of point defects $[18,19]$, but it cannot predict the change in the defect concentration under irradiation nor give information on the structure of the disordered region. Then, we have advanced the "disordered region model" into a "dislocation accumulation model" by assigning the disordered region to a dislocation dipole [26].

The dislocation accumulation model [26] is a kinetic model, taking into account the point and defect clusters shown in Figure 14. The reaction kinetics can be summarized as follows. An incident atom knocks a host atom, resulting in the formation of an interstitial and a vacancy (Frenkel pair). The fraction of atomic sites at which the Frenkel pair production can take place gradually decreases with increasing the concentration of vacancy and dislocation dipoles. Single interstitials move two-dimensionally parallel to the basal 


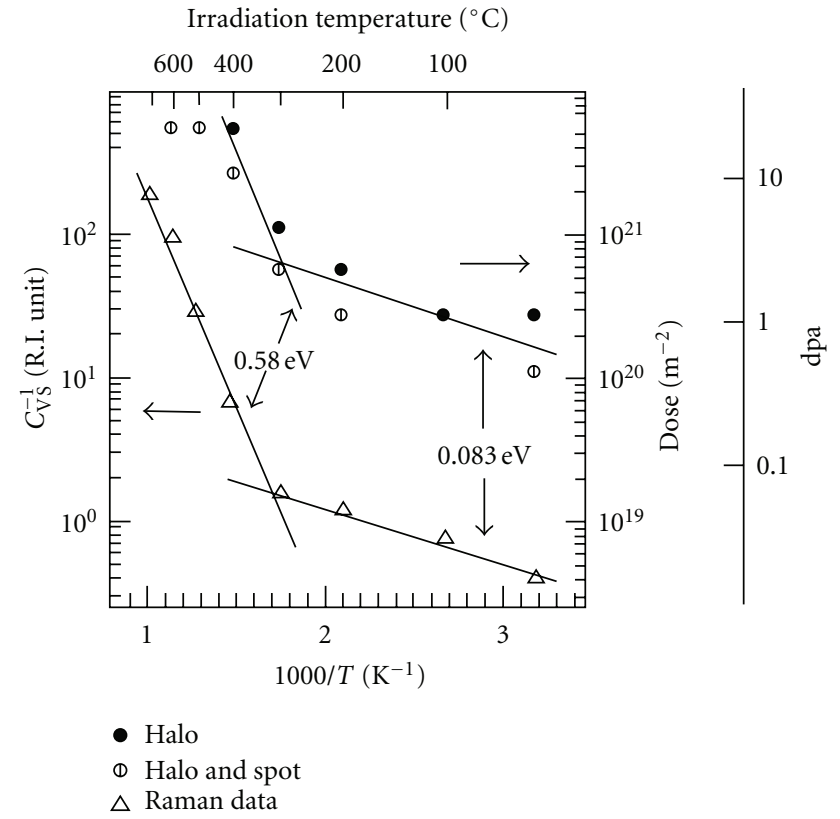

Figure 13: Arrhenius plot of $C_{V S}^{-1}$ determined by the fitting analysis. The values of $C_{V S}{ }^{-1}$ are expressed in R.I. Units. This plot corresponds to the critical dose of amorphization as expressed by (7). Two linear relations are clearly seen, giving activation energy of $0.083 \mathrm{eV}$ below $573 \mathrm{~K}$ and $0.58 \mathrm{eV}$ above $573 \mathrm{~K}$. The TEM results on the change of diffraction pattern from halo and spot to halo can be seen to give two similar linear relations.

plane. Other movements of interstitials and vacancies parallel to the basal plane and along the $c$-axis are neglected owing to the high activation energies. An interstitial in a recombination volume around a vacancy annihilates with the vacancy. A barrier exists for the mutual annihilation. A pair of interstitials is thermally stable once formed. We neglect the formation of interstitial clusters except for di-interstitials as we suppose that the interstitial clusters are not dominant for the total reaction kinetics at comparatively low temperatures. Divacancies formed by knock-ons, occurring at sites close to single vacancies, reconstruct to eliminate dangling bonds as shown in the left-hand side area of Figure 15. The reconstructed divacancies do not annihilate with interstitials and become the nucleus of the dislocation dipoles. Dislocation dipoles can grow by successive knock-ons close to the ends of dislocation dipoles as shown in the right-hand side area of Figure 15. The dislocation dipoles cannot annihilate with interstitials.

In Figure 16, the real-time change in the Raman intensity ratio of the original graphite peak and the defect peak, $I_{D} / I_{G}$, under ion irradiation at room temperature for two different production rates of the Frenkel pair $7.0 \times 10^{-7}$ and $6.7 \times 10^{-6} \mathrm{dpa} / \mathrm{s}[13]$ is compared with the dislocation accumulation model [26]. The experimental results of $I_{D} / I_{G}$ exhibit a square-root dependence against irradiation dose. To show the square-root dependence, the vacancy concentration $C_{V}$ should increase proportionally with the irradiation dose; that is, the rate of mutual annihilation of a vacancy and an interstitial should be negligibly small. By utilizing the proper value of $q=5.0 \times 10^{-4}$, which is a recombination probability relating to the barrier for mutual annihilation [26], the calculated results can be fitted to the experimental results as shown in Figure 16. The downward deviation from the square-root dose dependence for the upper result, which comes from the effect of mutual annihilation of interstitial and vacancy, can be simulated in the calculation. The dose range of Raman measurements done by Nakamura and Kitajima [13] corresponds to the initial stage where $C_{V}$ increases proportionally to the irradiation time; that is, $C_{V}=P t$. This is the reason why Raman intensity ratio exhibited the square-root dependence. To obtain the linear relationship, a very small value of the recombination probability between a vacancy and an interstitial, that is, a strong barrier for the mutual annihilation, is needed.

The existence of the strong barrier for the mutual annihilation was supported by another simulation [26] for the dose dependence of the $c$-axis expansion given by Koike and Pedraza [17] with the dislocation accumulation model. Figure 17 compares the dimensional change in highly oriented pyrolytic graphite (HOPG) along the $c$-axis during $300 \mathrm{keV}$ electron irradiation at room temperature with the results calculated by the present theory using several values of $q$. The dimensional change along the $c$-axis, $\Delta L_{c} / L_{c o}$, for the calculations is assumed to increase proportionally with the accumulation of di-interstitials. $J$ is a parameter representing the expansion rate per atom by the formation of di-interstitials. The agreement is quite acceptable with the values of $q=5.0 \times 10^{-4}$ and $J=4.2$ for the two stages of the initial fast elongation up to a dose of $0.2 \mathrm{dpa}$ and the subsequent linear elongation up to $4.0 \mathrm{dpa}$. The value of the expansion rate $J=4.2$ is acceptable in its order of magnitude compared to the case of no barrier $(q=1)$ where $J$ has to take an unacceptable high value of $10^{3}$ to accord with the experimental result of the dimensional change. Nevertheless, $J=4.2$ is larger than the value $J=1$ expected for the case when di-interstitials do not induce excess expansion. This may occur in cases where the di-interstitials effectively expand the lattice or dislocation dipoles induce buckling of the basal planes. One should note that the saturation of vacancy concentration assumed in the disordered-region model $[18,19]$ is modified to the peak formation of vacancy concentration in the dislocation accumulation model [26].

Utilizing (1) with a proper parameter of $k$, we can quantitatively deduce the concentration of vacancy $C_{V}$ from Raman intensity ratio in the case where most of defects are single vacancies. Previously, we estimated the value of $k$ as 0.0016 based on the in situ Raman measurement for $3 \mathrm{keV} \mathrm{He}^{+}$irradiation [13], where Raman intensity was corrected with the observed Raman intensity, as the damaged region is shallower than the region where Raman scattering is detectable. To obtain the precise value of $k$, it should be determined in the case where single vacancies are homogeneously distributed in the Raman detectable region. In this sense, the $25 \mathrm{keV} \mathrm{He}{ }^{+}$irradiation should be better than the $3 \mathrm{keV} \mathrm{He}^{+}$irradiation for the determination of $k$, because all Raman scattering comes from the damage region. 


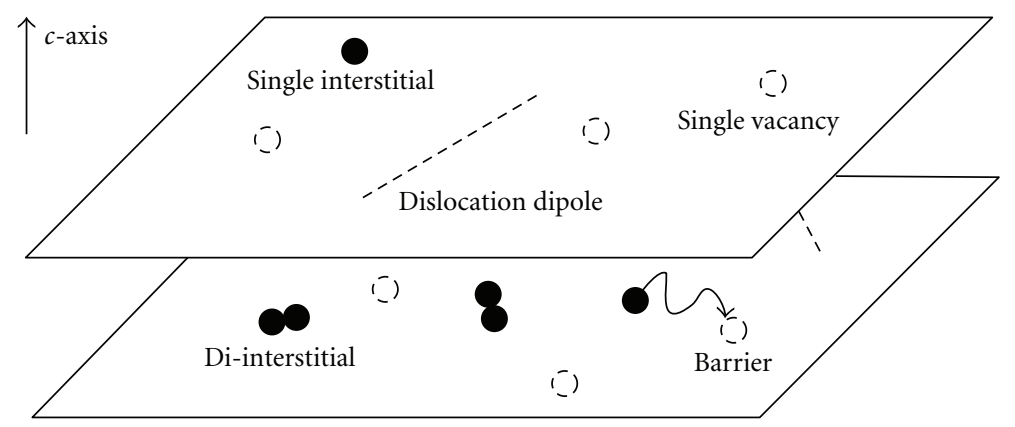

Figure 14: Point defects, defect clusters, and their reactions assumed in the dislocation dipole accumulation model. A recombination barrier exists between an interstitial and vacancy.

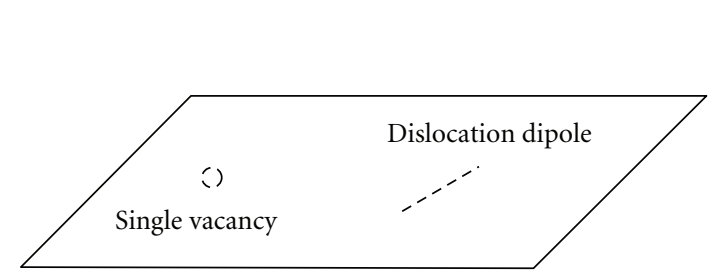

(a)

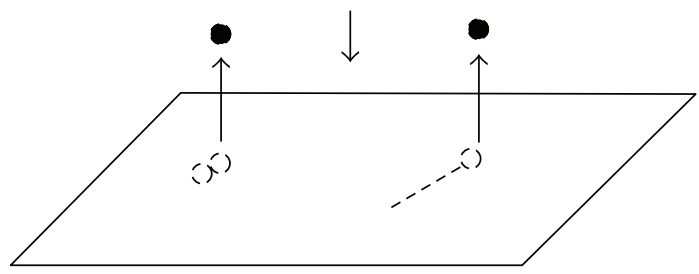

(b)

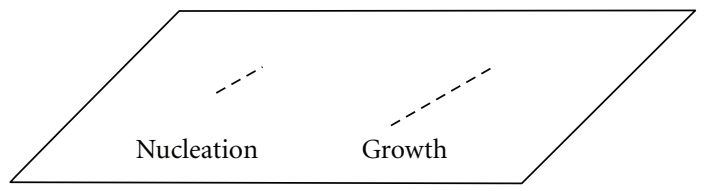

(c)

Figure 15: Nucleation and growth of dislocation dipole. Divacancies formed by knock-ons, occurring at sites close to single vacancies, reconstruct to eliminate dangling bonds as shown in the left-hand side area. The reconstructed divacancies are the nucleus of the dislocation dipoles. Dislocation dipoles can grow by successive knock-ons close to the ends of dislocation dipoles as shown in the right-hand side area.

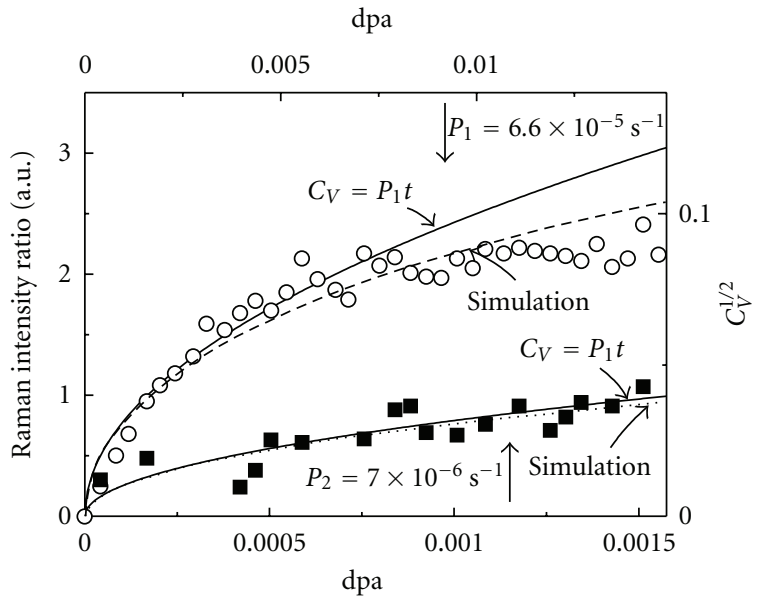

O Nakamura and Kitajima $\left(P_{1}\right)$

- Nakamura and Kitajima $\left(P_{2}\right)$

FIGURE 16: The real-time changes of Raman intensity ratio for $3 \mathrm{keV}$ $\mathrm{Ar}^{+}$ion irradiation at room temperature at fluxes of $7.0 \times 10^{-7}$ and $6.0 \times 10^{-6} \mathrm{dpa} \mathrm{s}^{-1}[13]$.
Taking the Raman intensity ratio of 1.5 and dpa of 0.05 at the dose of $0.13 \times 10^{20} \mathrm{He} / \mathrm{m}^{2}$ for the room temperature irradiation and assuming that all the vacancies formed by irradiation remained in the specimen, we obtained the value of $k$ as 0.022 . Then (1) can be expressed as

$$
C_{V}=0.022\left(\frac{I_{1355}}{I_{1580}}\right)^{2} \text {. }
$$

Validity of this equation would be discussed elsewhere.

3.4. Nucleation and Growth of Dislocation Dipole. Dislocation accumulation model assumes the nuclei of dislocation dipoles are divacancies and they grow by successive knockons close to the ends as shown in Figure 15. However, the model does not give any information on the structure of dislocation dipoles as simply shown by broken lines in Figures 14 and 15. So, here, we discuss the formation process of dislocation dipoles.

Figures 18(a) and 18(b) show two possibilities of the nucleation process of dislocation dipole, that is, the reconstruction of divacancies. The left one transforms to two 


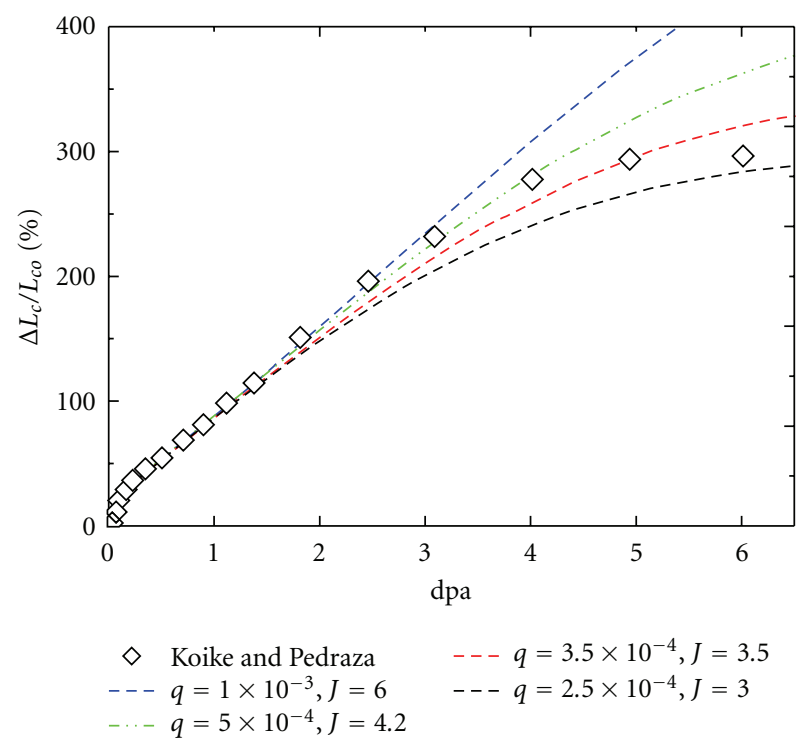

Figure 17: The dimensional change along the $c$-axis under $300 \mathrm{keV}$ electron irradiation at room temperature [17] and the simulation with the dislocation accumulation model with several values of $q$. The dimensional change is assumed to increase proportionally with the amount of di-interstitials accumulated between the basal planes. The values of $J$ correspond to the $c$-axis expansion ratio per atom of di-interstitials.

eightfold rings, sitting opposite to each other, and the right one to eightfold ring and two fivefold rings. The right one should be energetically favorable [27]. If knock-on occurs at sites denoted by dark spots in Figure 18(b), both of them would grow to the same structure consisting of eight-, seven, and fivefold rings as shown in Figure 18(c). Successive knock-ons at the ends of the dislocation dipoles induce their growth along the zigzag line.

A long dislocation dipole generated from nine vacancies (Figure 19(a)) is shown in Figure 19(b), where a vacancy line reconstructs to a dislocation dipole to make an energetically favorable structure by eliminating dangling bonds. In the reconstructed structure of Figure 19(b), one dangling bond remains at a site denoted by a circle. The dislocation dipole can grow by knock-on at the ends of dislocation dipole denoted by symbols in Figure 19(b). Neighboring eight- and sixfold rings at the left-hand side end of the dislocation dipole in Figure 19(b) transforms to neighboring seven- and fivefold rings in Figure 19(c) after a knock-on of an atom denoted by a circle. On the other hand, the neighboring seven- and fivefold rings at the right-hand side end of the dislocation dipole in Figure 19(b) can grow into the neighboring eight- and sixfold rings in Figure 19(c) after a knock-on of an atom denoted by a triangle. The two processes can repeat alternately, leading the growth of the dislocation dipole. The contraction in the basal plane along the direction denoted by the broken line in Figure 19(a) occurs. We should note that the dislocation dipole grows just at the ends where reconstruction can occur.
3.5. Accumulation of Dislocation Dipole and Broadening of Raman Spectra. The dislocation accumulation model depicts the following picture of the disordering process of graphite under irradiation, taking into account the accumulation of dislocation dipoles. In the dose range before approaching the peak of vacancy concentration, the longrange order of the graphite sheet along the $c$-axis and the sequence of the 6-member rings in the basal planes remain as the dislocation dipole formation is negligibly small. With increasing dose, the formation of dislocation dipoles by knock-on process becomes significant due to an increase of vacancy concentration. The accumulation of dislocation dipoles destroys the topological long-range order in the basal plane by introducing, rotation, and buckling of the graphite sheets, leading to the fragmentation of the basal planes. In the early stage of this process, the irradiation-induced disordered structure would still reflect the original layered structure of HOPG. In fact, in spite of the TEM diffraction pattern of graphite observed along the $c$-axis, which shows a halo when irradiated to a dose of about $1 \mathrm{dpa}$, the sequence of the basal planes still remains in the HREM images although the lattice image is broken up into small segments [12, 17, 30, 33]. The disordered graphite in this stage can be used as a raw material to generate amorphous diamond by shock compression [35]. In the highly accumulated state of the dislocation dipoles, nanocrystallization should become considerable and then the long-range order of the layered structure would disappear as shown by the HREM images of HOPG [17]. The formation of dislocation dipoles may play an important role in the generation of the concentric graphitic structure of "bucky onions" under electron irradiation [36], because the formation of the dislocation dipoles induces curvature of the graphitic layers.

One should remember that the remarkable broadening of Raman spectrum appears when disordering occurs under irradiation. This should be due to the life time broadening of phonons originated in the lattice distortion around the dislocation dipole. In contrary to this, the remarkable broadening does not occur in the initial stage of irradiation where single vacancies are dominantly formed. This suggests that the lattice distortion around single vacancy is not so strong to induce the remarkable life time broadening.

\section{Conclusion}

Raman spectral changes and associated TEM diffraction changes of highly oriented pyrolytic graphite (HOPG) under $25 \mathrm{keV} \mathrm{He}^{+}$and $20 \mathrm{keV} \mathrm{D}{ }^{+}$irradiation have been systematically investigated in terms of irradiation dose and temperature. Remarkable broadening of Raman peaks and appearance of halo rings in the TEM diffraction pattern indicate amorphization of the irradiated specimens after a critical dose. To explain the change of Raman spectra in terms of defect formation, we proposed "disorderedregion model" at first and then advanced the model into "dislocation accumulation model." These model calculations gave us important information on the critical dose of amorphization, a close relation between Raman intensity ratio and the vacancy concentration, an existence of barrier 


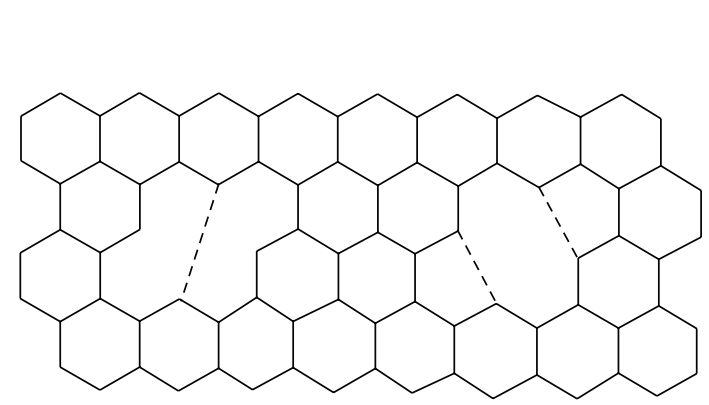

(a)

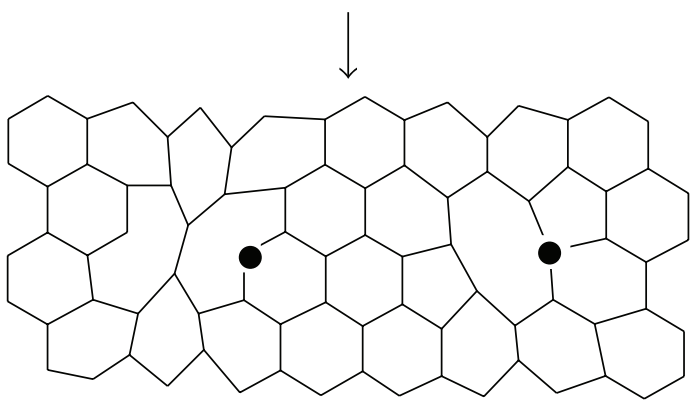

(b)

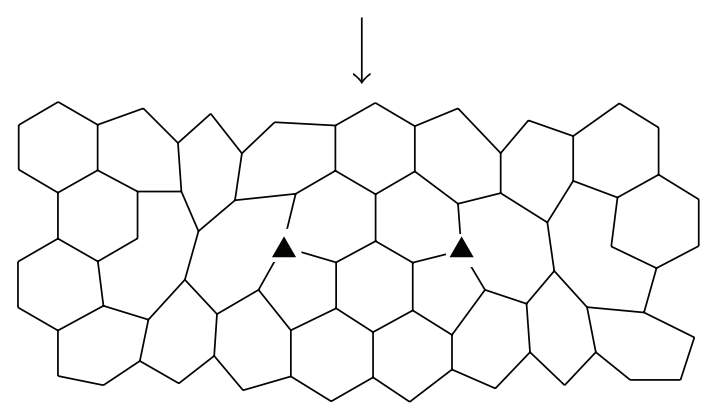

(c)

Figure 18: A possible nucleation process of dislocation dipole by the reconstruction of di-vacancy. The left one transforms to two eightfold rings, sitting opposite to each other, and the right one to eightfold ring and two fivefold rings. If knock-on occurs at sites denoted by circles in (b), both of them can transform to the same structure consisting of eightfold, sevenfold, and fivefold rings as shown in (c).

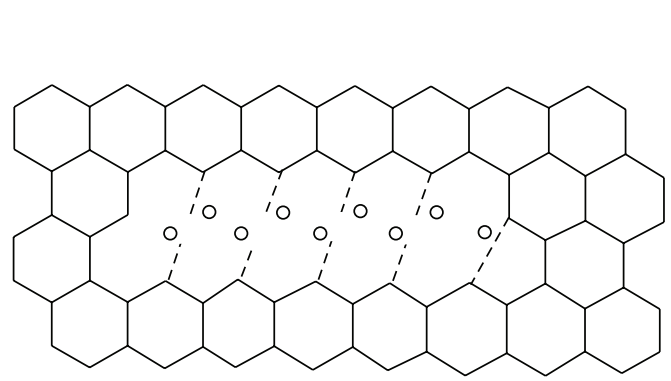

(a)

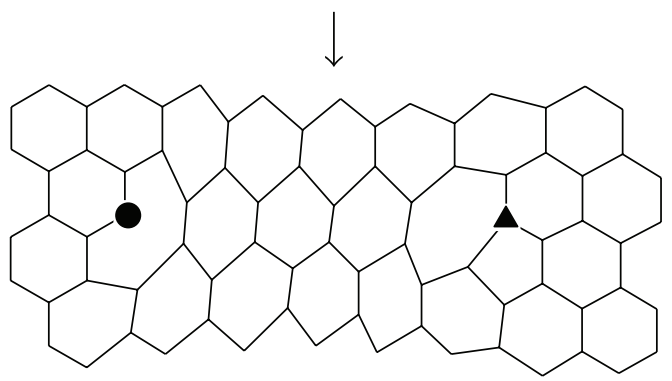

(b)

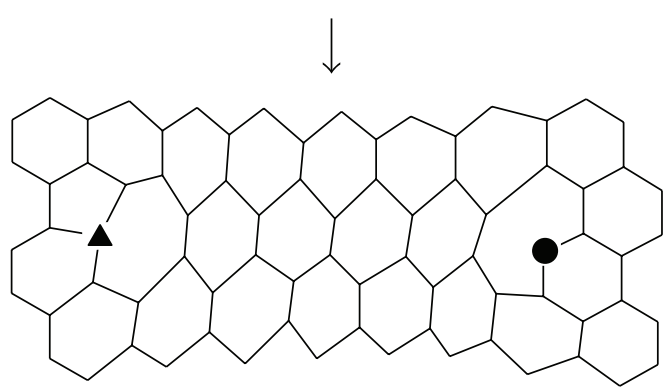

(c)

Figure 19: A long dislocation dipole generated from 9 vacancies (light spots). The dislocation dipole can grow by knock-on at the ends of dislocation dipole denoted by symbols in (b). Neighboring eightfold and sixfold rings at the left-hand side end of the dislocation dipole in (b) transforms to neighboring sevenfold and fivefold rings in (c) after a knock-on of an atom denoted by a circle. The neighboring sevenfold and fivefold rings can grow into the neighboring eightfold and sixfold rings as shown at the right-hand side area by knocking-on the atom denoted by a triangle. The contraction in the basal plane along the directions denoted by the broken lines in (a) occurs. 
on the mutual annihilation, and so forth. Thus, Raman spectroscopy can be a powerful tool for the analysis of point defect and defect clusters in irradiated graphite.

\section{Acknowledgments}

The author would like to thank many collaborators shown in the following references. This work was supported by the JSPS Grant-in-Aid (no. 23560787).

\section{References}

[1] L. Arnold, Windscale, 1957: Anatomy of a Nuclear Accident, Palgrave Macmillan, London, UK, 1995.

[2] W. N. Reynolds, Radiation Damage in Graphite, Pergamon, New York, NY, USA, 1965.

[3] P. A. Thrower, Chemistry and Physics of Carbon, vol. 5 of edited by P. L. Walker, Dekker, New York, NY, USA, 1969.

[4] P. A. Thrower and R. M. Mayer, "Point defects and self-diffusion in graphite," Physica Status Solidi A, vol. 47, no. 1, pp. 1137, 1978.

[5] B. T. Kelly, Physics of Graphite, Applied Science, London, UK, 1981.

[6] B. S. Elman, M. S. Dresselhaus, G. Dresselhaus, E. W. Maby, and H. Mazurek, "Raman scattering from ion-implanted graphite," Physical Review B, vol. 24, no. 2, pp. 1027-1034, 1981.

[7] T. Iwata, "Fine structure of Wigner energy release spectrum in neutron irradiated graphite," Journal of Nuclear Materials, vol. 133-134, pp. 361-364, 1985.

[8] K. Niwase, M. Sugimoto, T. Tanabe, and F. E. Fujita, "Electron microscope study of radiation damage in graphite produced by $\mathrm{D}^{+}$and $\mathrm{He}^{+}$bombardment," Journal of Nuclear Materials, vol. 155-157, no. 1, pp. 303-306, 1988.

[9] K. Niwase, K. Nakamura, I. Tanaka, Y. Miyamoto, and T. Tanabe, "Laser Raman microprobe analysis of the modified surface of $\mathrm{He}^{+}$-irradiated graphite," Journal of Nuclear Materials, vol. 179-181, no. 1, pp. 214-217, 1991.

[10] K. Niwase, T. Tanabe, and I. Tanaka, "Annealing experiment of ion-irradiated graphite by laser Raman spectroscopy," Journal of Nuclear Materials, vol. 191-194, pp. 335-339, 1992.

[11] K. Nakai, C. Kinoshita, and A. Matsunaga, "A study of amorphization and microstructural evolution of graphite under electron or ion irradiation," Ultramicroscopy, vol. 39, no. $1-4$, pp. 361-368, 1991.

[12] T. Tanabe, S. Muto, and K. Niwase, "On the mechanism of dimensional change of neutron irradiated graphite," Applied Physics Letters, vol. 61, no. 14, pp. 1638-1640, 1992.

[13] K. Nakamura and M. Kitajima, "Ion-irradiation effects on the phonon correlation length of graphite studied by Raman spectroscopy," Physical Review B, vol. 45, no. 1, pp. 78-82, 1992.

[14] M. S. Dresselhaus and R. Kalish, Ion Implantation in Diamond, Graphite and Related Materials, Springer, Berlin, Germany, 1992.

[15] K. Niwase and T. Tanabe, "Defect structure and amorphization of graphite irradiated by $\mathrm{D}^{+}$and $\mathrm{He}^{+}$," Materials Transactions, vol. 34, no. 11, pp. 1111-1121, 1993.

[16] B. T. Kelly, Materials Science and Technology, vol. 10, VCH, New York, NY, USA, 1994.
[17] J. Koike and D. F. Pedraza, "Dimensional changes in highly oriented pyrolytic graphite due to electron-irradiation," Journal of Materials Research, vol. 9, no. 7, pp. 1899-1907, 1994.

[18] K. Niwase, "Irradiation-induced amorphization of graphite," Physical Review B, vol. 52, no. 22, pp. 15785-15798, 1995.

[19] K. Niwase, "Erratum: irradiation-induced amorphization of graphite," Physical Review B, vol. 56, no. 9, pp. 5685-5685, 1997.

[20] T. Tanabe, T. Maruyama, M. Iseki, K. Niwase, and H. Atsumi, "Radiation damage of graphite: degradation of material parameters and defect structures," Fusion Engineering and Design, vol. 29, pp. 428-434, 1995.

[21] T. Tanabe, "Radiation damage of graphite-degradation of material parameters and defect structures," Physica Scripta T, vol. 64, pp. 7-16, 1996.

[22] H. Abe, H. Naramoto, A. Iwase, and C. Kinoshita, "Effect of damage cascades on the irradiation-induced amorphization in graphite," Nuclear Instruments and Methods in Physics Research, Section B, vol. 127-128, pp. 681-684, 1997.

[23] S. Muto and T. Tanabe, "Damage process in electron-irradiated graphite studied by transmission electron microscopy. I. High-resolution observation of highly graphitized carbon fibre," Philosophical Magazine A, vol. 76, no. 3, pp. 679-690, 1997.

[24] S. Muto, S. Horiuchi, and T. Tanabe, "Local structural order in electron-irradiated graphite studied by high-resolution highvoltage electron microscopy," Journal of Electron Microscopy, vol. 48, no. 6, pp. 767-776, 1999.

[25] T. Meguro, A. Hida, M. Suzuki et al., "Nanoscale modification of electronic states of graphite by highly charged Ar-ion irradiation," Journal of Vacuum Science and Technology B, vol. 19, no. 6, pp. 2745-2748, 2001.

[26] K. Niwase, "Irradiation-induced amorphization of graphite: a dislocation accumulation model," Philosophical Magazine Letters, vol. 82, no. 7, pp. 401-408, 2002.

[27] R. H. Telling, C. P. Ewels, A. A. El-Barbary, and M. I. Heggie, "Wigner defects bridge the graphite gap," Nature Materials, vol. 2, no. 5, pp. 333-337, 2003.

[28] A. Hashimoto, K. Suenaga, A. Gloter, K. Urita, and S. Iijima, "Direct evidence for atomic defects in graphene layers," Nature, vol. 430, no. 7002, pp. 870-873, 2004.

[29] K. Niwase, "Formation of dislocation dipoles in irradiated graphite," Materials Science and Engineering A, vol. 400-401, no. 1-2, pp. 101-104, 2005.

[30] A. Asthana, Y. Matsui, M. Yasuda, K. Kimoto, T. Iwata, and K. I. Ohshima, "Investigations on the structural disordering of neutron-irradiated highly oriented pyrolytic graphite by X-ray diffraction and electron microscopy," Journal of Applied Crystallography, vol. 38, no. 2, pp. 361-367, 2005.

[31] T. Hirai, J. Compan, K. Niwase, and J. Linke, "Laser Raman microprobe analysis of graphite exposed to edge plasma in the TEXTOR tokamak," Journal of Nuclear Materials, vol. 373, no. 1-3, pp. 119-122, 2008.

[32] T. Hirai, J. Compan, and K. Niwase, "Micro-Raman study on structure evolution of graphite exposed to intense thermal shock load," Advanced Materials Research, vol. 59, pp. 66-70, 2009.

[33] C. Karthik, J. Kane, D. P. Butt, W. E. Windes, and R. Ubic, "In situ transmission electron microscopy of electron-beam induced damage process in nuclear grade graphite," Journal of Nuclear Materials, vol. 412, no. 3, pp. 321-326, 2011. 
[34] F. Tuinstra and J. L. Koenig, "Raman spectroscopy of graphite," Journal of Chemical Physics, vol. 53, no. 3, pp. 1126-1130, 1970.

[35] K. Niwase, K. G. Nakamura, M. Yokoo, K. I. Kondo, and T. Iwata, "Pathway for the transformation from highly oriented pyrolytic graphite into amorphous diamond," Physical Review Letters, vol. 102, no. 11, Article ID 116803, 2009.

[36] D. Ugarte, "Formation mechanism of quasi-spherical carbon particles induced by electron bombardment," Chemical Physics Letters, vol. 207, no. 4-6, pp. 473-479, 1993. 


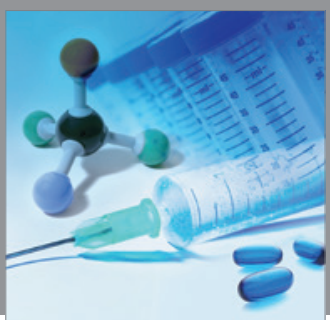

International Journal of

Medicinal Chemistry

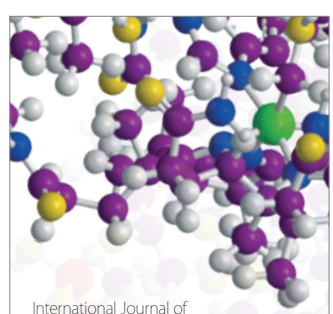

Carbohydrate Chemistry

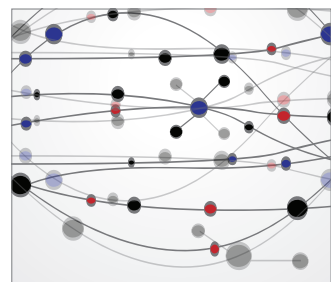

The Scientific World Journal
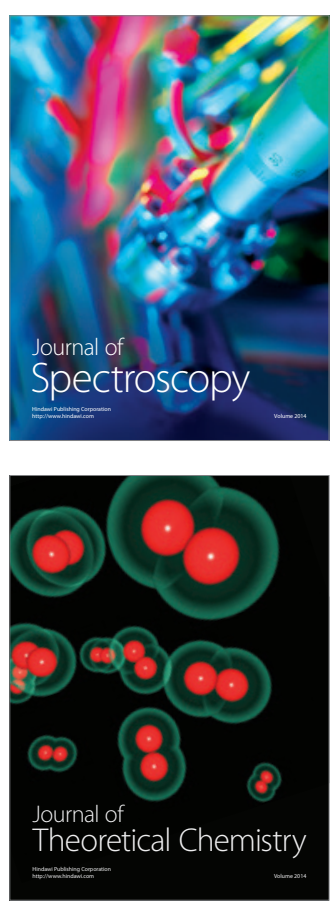
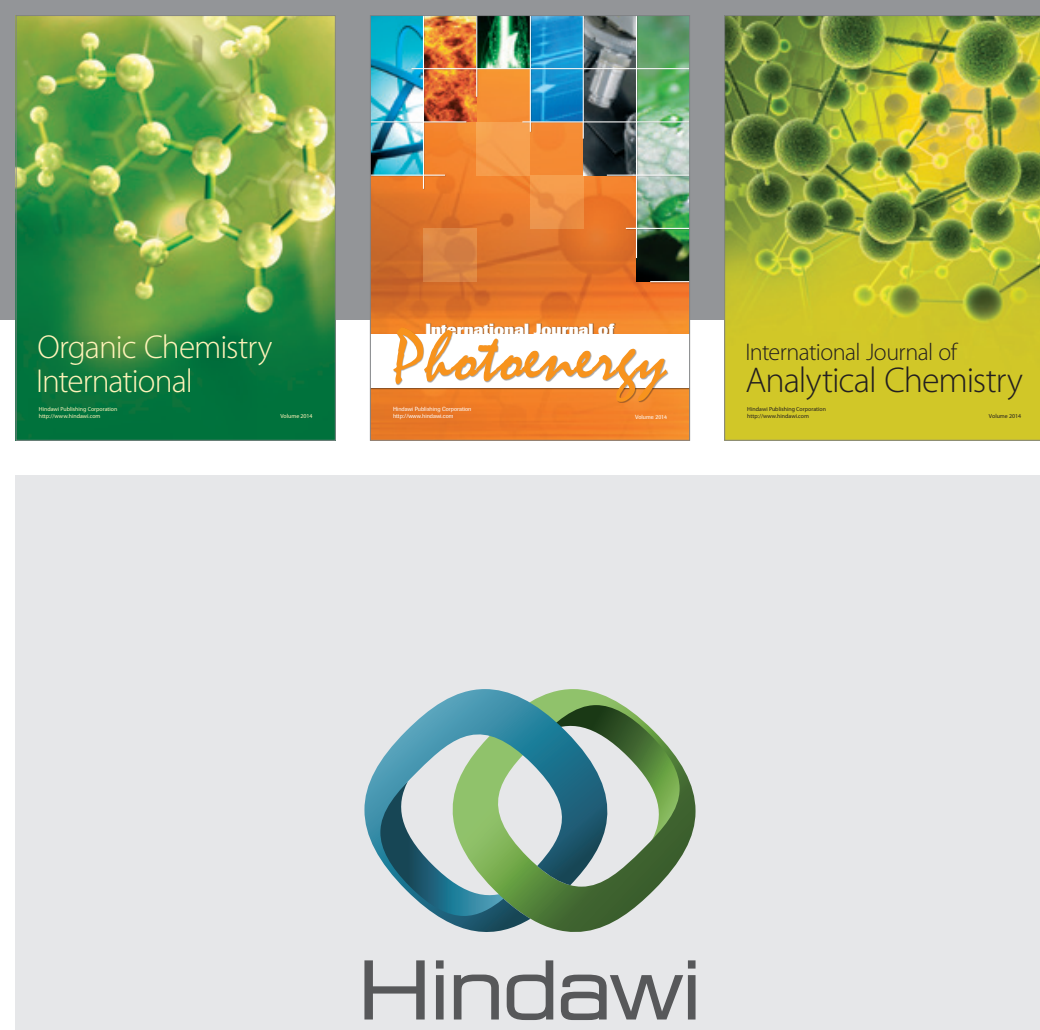

Submit your manuscripts at

http://www.hindawi.com
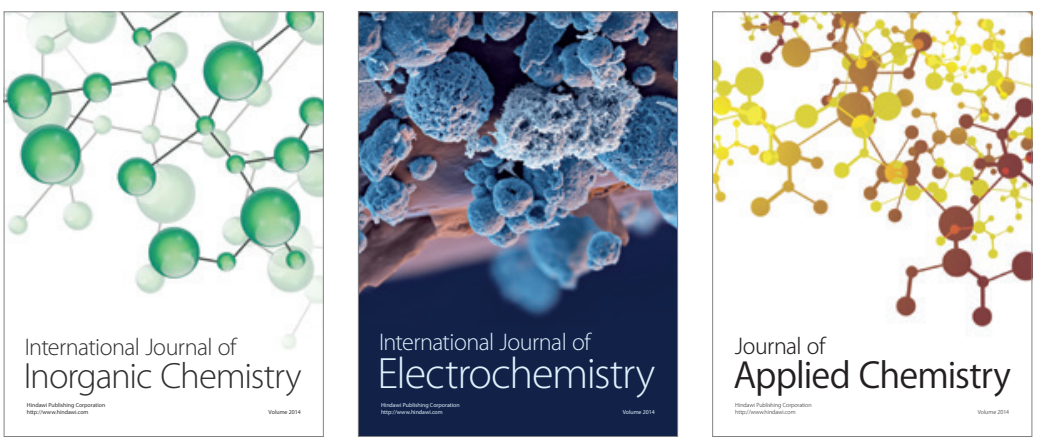

Journal of

Applied Chemistry
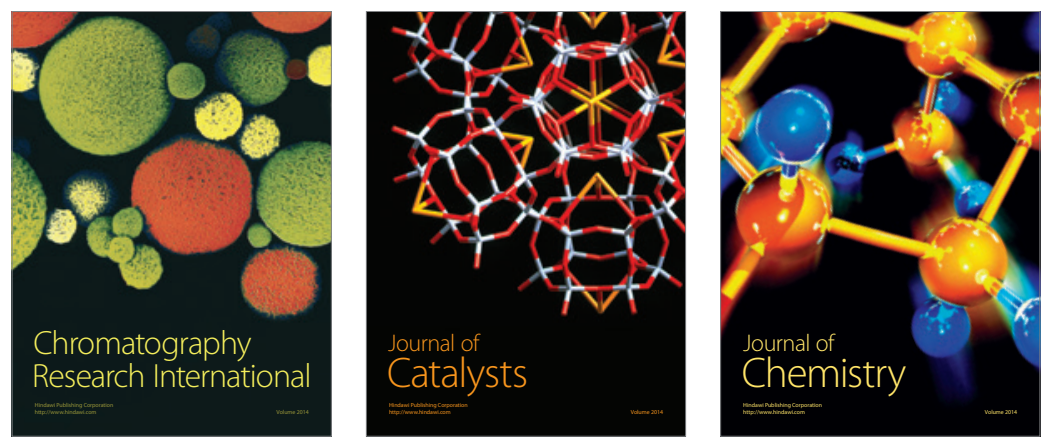
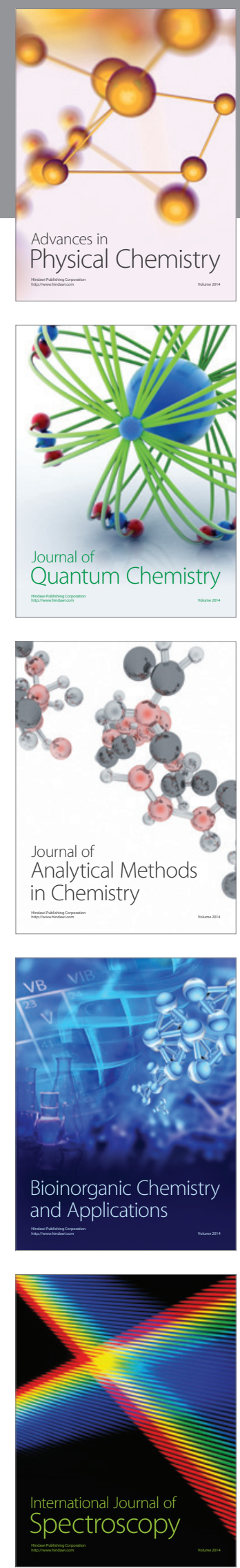